\title{
A Covariance Fitting Approach to Parametric Localization of Multiple Incoherently Distributed Sources
}

\author{
Shahram Shahbazpanahi, Member, IEEE, Shahrokh Valaee, Senior Member, IEEE, and \\ Alex B. Gershman, Senior Member, IEEE
}

\begin{abstract}
In this paper, a new algorithm for parametric localization of multiple incoherently distributed sources is presented. This algorithm is based on an approximation of the array covariance matrix using central and noncentral moments of the source angular power densities. Based on this approximation, a new computationally simple covariance fitting-based technique is proposed to estimate these moments. Then, the source parameters are obtained from the moment estimates. Compared with earlier algorithms, our technique has lower computational cost and obtains the parameter estimates in a closed form. In addition, it can be applied to scenarios with multiple sources that may have different angular power densities, while other known methods are not applicable to such scenarios.
\end{abstract}

Index Terms-Array processing, central and noncentral moments, covariance fitting, incoherently distributed sources, parametric localization.

\section{INTRODUCTION}

I $\mathrm{N}$ most applications of array processing, source localization methods are based on point source modeling, where it is assumed that the energy arriving at a sensor array originates from multiple point sources. In terms of direction finding, this means that the source energy is assumed to be concentrated at discrete angles which are referred to as the directions of arrival (DOAs). Based on this assumption, several high-resolution direction-finding methods have been proposed to estimate the source DOAs. MUSIC [1] and ESPRIT [2] are representative examples of such methods. However, in numerous applications such as sonar, radar, and wireless communications, signal scattering phenomena may cause angular spreading of the source energy [3]-[6]. Hence, in such cases, the distributed source model is more appropriate than the point source one [5].

In wireless communications, much attention has recently been paid to the use of the antenna array in order to exploit the

\footnotetext{
Manuscript received January 11, 2002; revised May 12, 2003. This work was supported in part by the Wolfgang Paul Award Program of the Alexander von Humboldt Foundation (Germany) and German Ministry of Education and Research, the Premier's Research Excellence Award Program of the Ministry of Energy, Science, and Technology (MEST) of Ontario, and the Natural Sciences and Engineering Research Council (NSERC) of Canada. The associate editor coordinating the review of this paper and approving it for publication was Dr. Karim Abed-Meraim.

S. Shahbazpanahi and A. B. Gershman are with the Department of Electrical and Computer Engineering, McMaster University, Hamilton, ON L8S 4K1 Canada.

S. Valaee is with the Edward S. Rogers Department of Electrical and Computer Engineering, University of Toronto, Toronto, ON M5S 3G4 Canada.

Digital Object Identifier 10.1109/TSP.2003.822352
}

spatial diversity [7]-[9] to improve the system coverage and capacity and reduce the effect of co-channel and intersymbol interference. In rural and suburban environments with a high base station, one of the main problems to be faced is the fast fading due to local scattering in the vicinity of the mobile [3]-[5]. As a consequence, the source is no longer viewed by the array as a point source as it represents a spatially distributed source with some central angle and angular spread. Practical measurements have shown that depending on the environment of the mobile, the base-mobile distance and the base station height, angular spreads up to $10^{\circ}$ are commonly encountered in practice [4], [6]. Note that depending on the relationship between the channel coherency time and the observation period, the sources can be viewed either as coherently distributed (CD) or incoherently distributed (ID). Indeed, if the channel coherency time is much smaller than the observation period, then the ID model is relevant. In the opposite case, the CD model or a partially coherent model can be used [10].

Several techniques have been proposed for distributed source localization. The first two attempts to generalize the signal and noise subspace concepts to distributed sources have been made in [11] and [12]. In these papers, extensions of the MUSIC estimator to the case of distributed sources are proposed. The first algorithm can be applied to both CD and ID sources, whereas the second one is able to treat ID source scenarios only. Both algorithms involve a two-dimensional (2-D) spectral search and, therefore, are computationally intensive.

In [13], it has been shown that apart from their high computational cost, the algorithms of [11] and [12] are severely restricted in the ID source case in that they do not provide consistent estimates of the source parameters. To overcome this drawback of the distributed source parameter estimator of (DSPE) of [11] and the dispersed signal parameter estimator (DISPARE) of [12], a class of weighted subspace fitting algorithms has been proposed [13] that gives consistent parameter estimates. However, the resulting algorithms have prohibitively high computational costs because they involve a multidimensional search over a highly nonlinear objective function.

In [14], a maximum likelihood (ML) algorithm has been proposed for localization of ID sources with Gaussian angular distributions. In this algorithm, the likelihood function is jointly maximized for all parameters of the model used. Similar to the techniques presented in [13], the computational complexity of this method remains prohibitively high as a multidimensional search over a highly nonlinear likelihood function is required. 
In [15] and [16], several techniques have been presented for localization of a uniformly incoherently distributed (UID) source. However, these algorithms cannot be applied to multiple source scenarios or any non-UID source scenario.

Another approach to the localization of distributed sources has been proposed in [5], where the Taylor series expansion has been used to derive an approximate model of a distributed source, which is referred to as the generalized array manifold (GAM) and is based on a linear combination of the array response vector and its derivatives. Using GAM, an algorithm has been proposed [5] to estimate the source spatial signature. The application of this algorithm is restricted to the case of a uniform linear array (ULA) and uniform CD sources.

Another interesting yet ad hoc approach to distributed source localization has been proposed in [17]. The authors have suggested an approximation of a distributed source by two point sources. Then, the central angle is estimated by averaging the angles obtained from the root-MUSIC polynomial, whereas the angular spread is obtained by using a lookup table. An essential shortcoming of this algorithm is that it is restricted to the single source case.

Recently, several covariance fitting techniques have been used to estimate the angular parameters of a distributed source [18]-[21]. For example, the so-called extended invariance principle (EXIP) algorithm [21] computes the central angle and angular spread parameters in an efficient way, using two successive one-dimensional (1-D) searches. However, the main shortcoming of these algorithms is that they are entirely based on the single source assumption and cannot be extended to the multiple source case.

In [22], the conventional beamformer has been applied to estimate the locations of distributed sources. It has been shown that the performance of this approach in the distributed source case can be far from the potentially achievable one, especially when the sources are closely spaced, their SNRs are high and essentially different, or the number of samples/sensors is large. However, in the case when the sources are widely spaced and have approximately the same power, the conventional beamformer is an excellent alternative to high-resolution methods because it combines robustness with computational efficiency and can be easily extended to arbitrary planar or volumetric arrays.

In [23], a subspace-based algorithm has been formulated that is applicable to the ID multiple source case. In this algorithm, the total least squares (TLS) ESPRIT approach is employed to estimate the source central angles. Then, the source angular spreads are estimated using the least squares (LS) covariance matrix fitting. However, as it will be demonstrated below, the performance of this algorithm may be unsatisfactory.

In the present paper, we develop a new algorithm for ID source localization. We use the Taylor series expansion of the array response vector to approximate the array covariance matrix using the central or noncentral moments of the source angular power densities. Based on this approximation, we propose a covariance fitting optimization to estimate these moments. We show that the source central angles and angular spreads can be obtained from the central and noncentral moments. Using the second central moment of the source angular power density as a measure of angular spread, we propose a simple way to estimate this parameter in the presence of sources with different angular power densities. The algorithm developed is applicable to multisource scenarios. Further, unlike the DSPE and DISPARE algorithms, it does not require any spectral search. As a result, the proposed method has lower computational cost than these techniques and outperforms the ESPRIT-based estimator presented in [23].

The paper is organized as follows. In Section II, we present our model for ID sources. In Section III, we show how the array covariance matrix can be approximated using the noncentral moments of the source angular power densities. Based on this approximation, a new estimation algorithm is developed in the same section. Computer simulation results are presented in Section IV, and conclusions are drawn in Section V.

\section{Signal Model}

Assume that stationary signals with the same central frequency $\omega_{0}$ impinge on an array of $p$ sensors from $q$ distributed narrowband far-field sources. The output of the $i$ th sensor of the array is given by

$$
x_{i}(t)=\sum_{m=1}^{q} \int_{-\pi / 2}^{\pi / 2} s_{m}\left(\theta, \psi_{m}, t\right) a_{i}(\theta) d \theta+n_{i}(t)
$$

where $s_{m}\left(\theta, \boldsymbol{\psi}_{m}, t\right)$ is the complex random time-varying angular distribution of the $m$ th source, $a_{i}(\theta)$ is the response of the $i$ th sensor to the unit energy source emitting from the direction $\theta, \boldsymbol{\psi}_{m}$ is the location parameter vector of the $m$ th source, and $n_{i}(t)$ is the additive zero-mean spatially white noise in the $i$ th sensor. Examples of the parameter vector $\boldsymbol{\psi}_{m}$ are the two angular bounds of a uniformly distributed source or the mean and standard deviation of a source with the Gaussian or Laplacian angular distributions. Equation (1) can be rewritten in the vector form as

$$
\boldsymbol{x}(t)=\sum_{m=1}^{q} \int_{-\pi / 2}^{\pi / 2} s_{m}\left(\theta, \boldsymbol{\psi}_{m}, t\right) \boldsymbol{a}(\theta) d \theta+\boldsymbol{n}(t)
$$

where

$$
\begin{aligned}
\boldsymbol{x}(t) & \triangleq\left[x_{1}(t), \ldots, x_{p}(t)\right]^{T} \\
\boldsymbol{a}(\theta) & \triangleq\left[a_{1}(\theta), \ldots, a_{p}(\theta)\right]^{T} \\
\boldsymbol{n}(t) & \triangleq\left[n_{1}(t), \ldots, n_{p}(t)\right]^{T}
\end{aligned}
$$

are the array observation, array response, and sensor noise vectors, respectively, and $(\cdot)^{T}$ denotes the transpose. Assuming that the sources and noise are uncorrelated, the covariance matrix can be written as

$$
\begin{aligned}
\boldsymbol{R}_{x x} & \triangleq \mathrm{E}\left\{\boldsymbol{x}(t) \boldsymbol{x}^{H}(t)\right\} \\
& =\sum_{m=1}^{q} \sum_{n=1}^{q} \int_{-\pi / 2}^{\pi / 2} \int_{-\pi / 2}^{\pi / 2} p_{m n}\left(\theta, \theta^{\prime} ; \boldsymbol{\psi}_{m}, \boldsymbol{\psi}_{n}\right) \boldsymbol{a}(\theta) \boldsymbol{a}^{H}\left(\theta^{\prime}\right) d \theta d \theta^{\prime} \\
& +\sigma^{2} \boldsymbol{I}
\end{aligned}
$$

where $\sigma^{2}$ is the unknown noise power, $\boldsymbol{I}$ is the identity matrix, $\mathrm{E}\{\cdot\}$ is the statistical expectation operator, and $(\cdot)^{H}$ denotes the Hermitian transpose. The function

$$
p_{m n}\left(\theta, \theta^{\prime} ; \boldsymbol{\psi}_{m}, \boldsymbol{\psi}_{n}\right) \triangleq \mathrm{E}\left\{s_{m}\left(\theta, \boldsymbol{\psi}_{m}, t\right) s_{n}^{*}\left(\theta^{\prime}, \boldsymbol{\psi}_{n}, t\right)\right\}
$$


is termed as the angular cross-correlation kernel, where $(\cdot)^{*}$ stands for the complex conjugate.

Throughout the paper, we will consider the ID source model. ${ }^{1}$ As a consequence, the source is no longer viewed by the array as a point source as it becomes a spatially distributed source with some central angle and angular spread.

A distributed source is said to be ID if its components arriving from different directions are uncorrelated. That is, for the $m$ th source, we have

$$
p_{m m}\left(\theta, \theta^{\prime} ; \boldsymbol{\psi}_{m}, \boldsymbol{\psi}_{m}\right)=\sigma_{m}^{2} \rho_{m}\left(\theta, \boldsymbol{\psi}_{m}\right) \delta\left(\theta-\theta^{\prime}\right)
$$

where $\delta\left(\theta-\theta^{\prime}\right)$ is the Dirac delta-function, $\sigma_{m}^{2}$ is the power of the $m$ th source, and $\rho_{m}\left(\theta, \boldsymbol{\psi}_{m}\right)$ is its normalized angular power density. The index $m$ in $\rho_{m}\left(\theta, \boldsymbol{\psi}_{m}\right)$ is used to emphasize that the sources can have different parameterized angular power densities. Note that

$$
\int_{-\pi / 2}^{\pi / 2} \rho_{m}\left(\theta, \boldsymbol{\psi}_{m}\right) d \theta=1, \quad m=1,2, \ldots, q .
$$

Let us assume that all distributed sources are mutually uncorrelated. Then, we can rewrite (7) as

$$
p_{m n}\left(\theta, \theta^{\prime} ; \boldsymbol{\psi}_{m}, \boldsymbol{\psi}_{n}\right)=\sigma_{m}^{2} \rho_{m}\left(\theta, \boldsymbol{\psi}_{m}\right) \delta\left(\theta-\theta^{\prime}\right) \delta_{m n}
$$

where $\delta_{m n}$ is the Kronecker delta. Using (10), we can rewrite (6) as

$$
\boldsymbol{R}_{x x}=\sum_{m=1}^{q} \int_{-\pi / 2}^{\pi / 2} \sigma_{m}^{2} \rho_{m}\left(\theta, \boldsymbol{\psi}_{m}\right) \boldsymbol{a}(\theta) \boldsymbol{a}^{H}(\theta) d \theta+\sigma^{2} \boldsymbol{I} .
$$

For further convenience, let us define the central angle of the $m$ th source as the mass center of the source angular power density

$$
\theta_{0 m} \triangleq \frac{\int_{-\pi / 2}^{\pi / 2} \theta \rho_{m}\left(\theta, \boldsymbol{\psi}_{m}\right) d \theta}{\int_{-\pi / 2}^{\pi / 2} \rho_{m}\left(\theta, \boldsymbol{\psi}_{m}\right) d \theta}=\int_{-\pi / 2}^{\pi / 2} \theta \rho_{m}\left(\theta, \boldsymbol{\psi}_{m}\right) d \theta
$$

The source central angles form the vector

$$
\boldsymbol{\theta}_{0}=\left[\theta_{01}, \theta_{02}, \ldots, \theta_{0 q}\right]^{T} .
$$

Next, let us define the $n$th noncentral moment of the angular power density of the $m$ th source around $\tilde{\theta}_{0 m}$ as

$$
M_{n m}\left(\tilde{\theta}_{0 m}\right) \triangleq \int_{-\pi / 2}^{\pi / 2}\left(\theta-\tilde{\theta}_{0 m}\right)^{n} \rho_{m}\left(\theta, \boldsymbol{\psi}_{m}\right) d \theta
$$

where $\tilde{\theta}_{0 m}$ is an arbitrary angle, and for the sake of brevity, we use the notation where the dependence of $M_{n m}$ on $\boldsymbol{\psi}_{m}$ is not shown explicitly.

In what follows, $\tilde{\theta}_{0 m}$ will be viewed as a coarse initialization of the true central angle $\theta_{0 m}$. If $\tilde{\theta}_{0 m}=\theta_{0 m}$, then $M_{n m}\left(\tilde{\theta}_{0 m}\right)$ becomes the $n$th central moment $M_{n m}\left(\theta_{0 m}\right)$ of the $m$ th source angular power density. The following lemma is of key importance for our subsequent derivations.

\footnotetext{
${ }^{1}$ The assumption of uncorrelated ID sources has been theoretically and experimentally shown to be relevant in wireless communications in the case of rural and suburban environments with a high base station [3]-[5].
}

Lemma 1: For the $m$ th source, the value of the first noncentral moment around an arbitrary angle $\tilde{\theta}_{0 m}$ determines the deviation of $\tilde{\theta}_{0 m}$ with respect to the central angle $\theta_{0 m}$.

Proof: Using (14), we have

$$
\begin{aligned}
M_{1 m}\left(\tilde{\theta}_{0 m}\right) & =\int_{-\pi / 2}^{\pi / 2}\left(\theta-\tilde{\theta}_{0 m}\right) \rho_{m}\left(\theta, \boldsymbol{\psi}_{m}\right) d \theta \\
& =\int_{-\pi / 2}^{\pi / 2} \theta \rho_{m}\left(\theta, \boldsymbol{\psi}_{m}\right) d \theta-\tilde{\theta}_{0 m} \int_{-\pi / 2}^{\pi / 2} \rho_{m}\left(\theta, \boldsymbol{\psi}_{m}\right) d \theta \\
& =\theta_{0 m}-\tilde{\theta}_{0 m}
\end{aligned}
$$

where the last row of (15) follows from (9) and (12).

From Lemma 1, we conclude that given some estimate for the first noncentral moment, we are able to estimate the source central angle from (15).

In what follows, we assume that the angular distribution of each source is determined by the normalized angular power density, which is a non-negative function parameterized by two parameters: the central angle and the angular spread. We also assume that different sources may have different shapes of their angular distribution function. However, for each source, we assume that we know the shape of the angular power density function $^{2}$ (for example, we know whether it is Gaussian, Laplacian, or uniform), but we do not know the parameters of this shape that have to be estimated.

\section{Covariance Fitting}

In this section, we show that the array covariance matrix can be approximated using a few noncentral moments of the source angular power densities. Then, we derive a new computationally simple covariance fitting-based direction finding algorithm. Note that the covariance fitting scheme (sometimes referred to as the covariance matching approach) has been used earlier for distributed source localization (see [14], [18]-[20], [25]-[28], and references therein).

Let us define

$$
\boldsymbol{C}(\theta) \triangleq \boldsymbol{a}(\theta) \boldsymbol{a}^{H}(\theta) .
$$

Consider an $I$-term Taylor series approximation of $\boldsymbol{C}(\theta)$ around $\tilde{\theta}_{0 m}$

$$
\boldsymbol{C}(\theta) \simeq \sum_{i=0}^{I-1}\left(\theta-\tilde{\theta}_{0 m}\right)^{i} \boldsymbol{C}_{i m}\left(\tilde{\theta}_{0 m}\right)
$$

where

$$
\left.\boldsymbol{C}_{i m}\left(\tilde{\theta}_{0 m}\right) \triangleq \frac{1}{i !} \frac{\partial^{i} \boldsymbol{C}(\theta)}{\partial \theta^{i}}\right|_{\theta=\tilde{\theta}_{0 m}} .
$$

The Taylor series approximation is a widely accepted approach that is used for characterization of spread or moving sources in array processing and adaptive beamforming (for example, see [5], [29]-[31], and references therein). Note that there is a tradeoff between the quality of source approximation and sensitivity to array calibration errors. Indeed, we are able to ap-

\footnotetext{
${ }^{2}$ It should be noted that the estimation of the source angular power density is a very difficult task [24]. However, the information about the form of this function can be obtained from measurement campaigns [4], [6]. Furthermore, the knowledge of the shape of the angular power density is not so critical for our approach (see the end of Section III).
} 
proximate the matrix $\boldsymbol{C}(\theta)$ increasingly more accurately by increasing the parameter $I$ in (17), but the sensitivity to array calibration errors will also increase due to the presence of higher order derivatives. Therefore, in practical situations, $I$ should not be much larger than one.

Inserting (17) into (11), we have the following approximation of the covariance matrix (11):

$$
\boldsymbol{R}_{x x} \simeq \tilde{\boldsymbol{R}}+\sigma^{2} \boldsymbol{I}
$$

where

$$
\tilde{\boldsymbol{R}}=\sum_{m=1}^{q} \sum_{r=0}^{I-1} \sigma_{m}^{2} M_{r m}\left(\tilde{\theta}_{0 m}\right) \boldsymbol{C}_{r m}\left(\tilde{\theta}_{0 m}\right) .
$$

Equation (19) represents an approximation of the exact covariance matrix (6) using $q I+1$ matrices. We will now use this approximation to formulate our direction-finding algorithm.

Using the LS criterion and (19) and (20), let us minimize the function

$$
\begin{aligned}
f\left(\boldsymbol{m}\left(\tilde{\boldsymbol{\theta}}_{0}\right), \tilde{\boldsymbol{\theta}}_{0}\right) & \triangleq\left\|\hat{\boldsymbol{R}}_{x x}-\tilde{\boldsymbol{R}}-\sigma^{2} \boldsymbol{I}\right\|^{2} \\
& =\| \hat{\boldsymbol{R}}_{x x}-\sum_{m=1}^{q} \sum_{r=0}^{I-1} \sigma_{m}^{2} M_{r m}\left(\tilde{\theta}_{0 m}\right) \boldsymbol{C}_{r m}\left(\tilde{\theta}_{0 m}\right) \\
& -\sigma^{2} \boldsymbol{I} \|^{2}
\end{aligned}
$$

where

$$
\hat{\boldsymbol{R}}_{x x}=\frac{1}{N} \sum_{t=1}^{N} \boldsymbol{x}(t) \boldsymbol{x}^{H}(t)
$$

is the sample covariance matrix, and the vectors

$$
\begin{aligned}
\tilde{\boldsymbol{\theta}}_{0} & \triangleq\left[\tilde{\theta}_{01}, \tilde{\theta}_{02}, \ldots, \tilde{\theta}_{0 q}\right]^{T} \\
\boldsymbol{m}\left(\tilde{\boldsymbol{\theta}}_{0}\right) & \triangleq\left[\boldsymbol{m}_{1}^{T}\left(\tilde{\theta}_{01}\right), \boldsymbol{m}_{2}^{T}\left(\tilde{\theta}_{02}\right), \ldots, \boldsymbol{m}_{q}^{T}\left(\tilde{\theta}_{0 q}\right), \sigma^{2}\right]^{T} \\
\boldsymbol{m}_{m}\left(\tilde{\theta}_{0 m}\right) & \triangleq \sigma_{m}^{2}\left[1, M_{1 m}\left(\tilde{\theta}_{0 m}\right), \ldots, M_{(I-1) m}\left(\tilde{\theta}_{0 m}\right)\right]^{T}
\end{aligned}
$$

contain the model parameters.

Assuming some initial value $\tilde{\boldsymbol{\theta}}_{0}$ for the vector $\boldsymbol{\theta}_{0}$, we find the estimate of (24) as

$$
\begin{aligned}
\hat{\boldsymbol{m}}\left(\tilde{\boldsymbol{\theta}}_{0}\right) & =\arg \min _{\boldsymbol{m}}\left\|\hat{\boldsymbol{R}}_{x x}-\tilde{\boldsymbol{R}}-\sigma^{2} \boldsymbol{I}\right\|^{2} \\
& =\arg \min _{\boldsymbol{m}} \operatorname{tr}\left\{\left(\hat{\boldsymbol{R}}_{x x}-\left(\tilde{\boldsymbol{R}}+\sigma^{2} \boldsymbol{I}\right)\right)^{2}\right\}
\end{aligned}
$$

where $\operatorname{tr}\{\cdot\}$ denotes the trace operator.

Differentiating $f\left(\boldsymbol{m}\left(\tilde{\boldsymbol{\theta}}_{0}\right), \tilde{\boldsymbol{\theta}}_{0}\right)$ with respect to the $i$ th $(i=1,2, \ldots, q I)$ element of $\boldsymbol{m}\left(\tilde{\boldsymbol{\theta}}_{0}\right)$, we get

$$
\begin{aligned}
f_{i}^{\prime} & \triangleq \frac{\partial f\left(\boldsymbol{m}\left(\tilde{\boldsymbol{\theta}}_{0}\right), \tilde{\boldsymbol{\theta}}_{0}\right)}{\partial\left[\boldsymbol{m}\left(\tilde{\boldsymbol{\theta}}_{0}\right)\right]_{i}} \\
& =\frac{\partial}{\partial\left[\boldsymbol{m}\left(\tilde{\boldsymbol{\theta}}_{0}\right)\right]_{i}}\left(\operatorname{tr}\left\{\left(\hat{\boldsymbol{R}}_{x x}-\left(\tilde{\boldsymbol{R}}+\sigma^{2} \boldsymbol{I}\right)\right)^{2}\right\}\right) \\
& =-2 \operatorname{tr}\left\{\left(\hat{\boldsymbol{R}}_{x x}-\left(\tilde{\boldsymbol{R}}+\sigma^{2} \boldsymbol{I}\right)\right) \boldsymbol{C}_{k l}\left(\tilde{\theta}_{0 l}\right)\right\} \\
& =2 \sum_{m=1 r=0}^{q-1} \sum_{r=1}^{I-} \operatorname{tr}\left\{\boldsymbol{C}_{r m}\left(\tilde{\theta}_{0 m}\right) \boldsymbol{C}_{k l}\left(\tilde{\theta}_{0 l}\right)\right\}\left[\boldsymbol{m}\left(\tilde{\boldsymbol{\theta}}_{0}\right)\right]_{(m-1) I+r+1} \\
& +2 \operatorname{tr}\left\{\boldsymbol{C}_{k l}\left(\tilde{\theta}_{0 l}\right)\right\}\left[\boldsymbol{m}\left(\tilde{\boldsymbol{\theta}}_{0}\right)\right]_{q I+1} \\
& -2 \operatorname{tr}\left\{\hat{\boldsymbol{R}}_{x x} \boldsymbol{C}_{k l}\left(\tilde{\theta}_{0 l}\right)\right\}
\end{aligned}
$$

where (20), (24), and (25) are used, and $[\cdot]_{i}$ denotes the $i$ th element of a vector. Here, it is assumed that $i=(l-1) I+k+1$ $(0 \leq k<I, 1 \leq l \leq q)$. Differentiating $f\left(\boldsymbol{m}\left(\tilde{\boldsymbol{\theta}}_{0}\right), \tilde{\boldsymbol{\theta}}_{0}\right)$ with respect to the $(q I+1)$ st element of $\boldsymbol{m}\left(\tilde{\boldsymbol{\theta}}_{0}\right)$, we have

$$
\begin{aligned}
f_{q I+1}^{\prime} & \triangleq \frac{\partial f\left(\boldsymbol{m}\left(\tilde{\boldsymbol{\theta}}_{0}\right), \tilde{\boldsymbol{\theta}}_{0}\right)}{\partial\left[\boldsymbol{m}\left(\tilde{\boldsymbol{\theta}}_{0}\right)\right]_{q I+1}} \\
& =\frac{\partial}{\partial\left[\boldsymbol{m}\left(\tilde{\boldsymbol{\theta}}_{0}\right)\right]_{q I+1}}\left(\operatorname{tr}\left\{\left(\hat{\boldsymbol{R}}_{x x}-\left(\tilde{\boldsymbol{R}}+\sigma^{2} \boldsymbol{I}\right)\right)^{2}\right\}\right) \\
& =-2 \operatorname{tr}\left\{\left(\hat{\boldsymbol{R}}_{x x}-\left(\tilde{\boldsymbol{R}}+\sigma^{2} \boldsymbol{I}\right)\right)\right\} \\
& =2 \sum_{m=1}^{q} \sum_{r=0}^{I-1} \operatorname{tr}\left\{\boldsymbol{C}_{r m}\left(\tilde{\theta}_{0 m}\right)\right\}\left[\boldsymbol{m}\left(\tilde{\boldsymbol{\theta}}_{0}\right)\right]_{(m-1) I+r+1} \\
& +2 p\left[\boldsymbol{m}\left(\tilde{\boldsymbol{\theta}}_{0}\right)\right]_{q I+1}-2 \operatorname{tr}\left\{\hat{\boldsymbol{R}}_{x x}\right\}
\end{aligned}
$$

Then, equating (27) and (28) to zero and rewriting these equations in the vector form as

$$
\left[f_{1}^{\prime}, f_{2}^{\prime}, \ldots, f_{q I+1}^{\prime}\right]^{T}=\mathbf{0}
$$

after straightforward manipulations, we have

$$
\boldsymbol{Q}\left(\tilde{\boldsymbol{\theta}}_{0}\right) \boldsymbol{m}\left(\tilde{\boldsymbol{\theta}}_{0}\right)=\boldsymbol{p}\left(\tilde{\boldsymbol{\theta}}_{0}\right)
$$

where

$$
\begin{aligned}
& {\left[\boldsymbol{Q}\left(\tilde{\boldsymbol{\theta}}_{0}\right)\right]_{i j} } \triangleq \operatorname{tr}\left\{\boldsymbol{C}_{k l}\left(\tilde{\theta}_{0 l}\right) \boldsymbol{C}_{r m}\left(\tilde{\theta}_{0 m}\right)\right\} \\
& {\left[\boldsymbol{p}\left(\tilde{\boldsymbol{\theta}}_{0}\right)\right]_{i} \triangleq \operatorname{tr}\left\{\boldsymbol{C}_{k l}\left(\tilde{\theta}_{0 l}\right) \hat{\boldsymbol{R}}_{x x}\right\} }
\end{aligned}
$$

for $i=(l-1) I+k+1, j=(m-1) I+r+1,1 \leq l, m \leq q$, $0 \leq k, r<I$, and

$$
\begin{aligned}
{\left[\boldsymbol{Q}\left(\tilde{\boldsymbol{\theta}}_{0}\right)\right]_{q I+1, j} } & \triangleq \operatorname{tr}\left\{\boldsymbol{C}_{r m}\left(\tilde{\theta}_{0 m}\right)\right\} \\
{\left[\boldsymbol{Q}\left(\tilde{\boldsymbol{\theta}}_{0}\right)\right]_{j, q I+1} } & \triangleq \operatorname{tr}\left\{\boldsymbol{C}_{r m}\left(\tilde{\theta}_{0 m}\right)\right\} \\
{\left[\boldsymbol{Q}\left(\tilde{\boldsymbol{\theta}}_{0}\right)\right]_{q I+1, q I+1} } & \triangleq p \\
{\left[\boldsymbol{p}\left(\tilde{\boldsymbol{\theta}}_{0}\right)\right]_{q I+1} } & \triangleq \operatorname{tr}\left\{\hat{\boldsymbol{R}}_{x x}\right\} .
\end{aligned}
$$

The solution to (30) is given by ${ }^{3}$

$$
\hat{\boldsymbol{m}}\left(\tilde{\boldsymbol{\theta}}_{0}\right)=Q^{-1}\left(\tilde{\boldsymbol{\theta}}_{0}\right) \boldsymbol{p}\left(\tilde{\boldsymbol{\theta}}_{0}\right)
$$

Using (37), we estimate the noncentral moments, and then, using Lemma 1, the central angles can be estimated as

$$
\hat{\theta}_{0 m}=\hat{M}_{1 m}\left(\tilde{\theta}_{0 m}\right)+\tilde{\theta}_{0 m}
$$

where $\hat{M}_{1 m}\left(\tilde{\theta}_{0 m}\right)$ is the first estimated noncentral moment of the angular density of the $m$ th source.

Note that $\tilde{\theta}_{0 m}$ is an arbitrary angle that should be chosen sufficiently close to $\theta_{0 m}$. In practice, most of the source energy is concentrated around the central angles, and if the difference between $\tilde{\theta}_{0 m}$ and $\theta_{0 m}$ is large, then the accuracy of the covariance

${ }^{3}$ If the matrix $\boldsymbol{Q}\left(\tilde{\boldsymbol{\theta}}_{0}\right)$ is singular or ill-conditioned, one can replace its inverse by pseudoinverse. However, note that in our simulations (based on multiple simulation runs, see below) there was no single run where this matrix became singular or ill-conditioned. 
approximation (20) will be low, and this will increase the estimation errors. Therefore, it is important to select $\tilde{\theta}_{0 m}$ as close as possible to $\theta_{0 m}$ to maintain the estimation errors reasonably small.

When the central angles are estimated, we can obtain the estimates of the central moments $\hat{M}_{n m}\left(\hat{\theta}_{0 m}\right)(m=1,2, \ldots, q)$ by means of solving the system (30) again with $\tilde{\theta}_{0 m}$ replaced by $\hat{\theta}_{0 m}$. Hence, the estimation algorithm should involve two stages. In the first stage, the noncentral moments and, consequently, the central angles are estimated, whereas in the second stage, the central moments can be obtained using previously estimated central angles.

According to our assumptions, the angular power density of each source is determined by its central angle and angular spread. It is clear that all central moments are related to the second parameter. The functional form of the angular power density determines this relationship. For instance, for a UID source with the central angle $\theta_{0 m}$ and the angular spread $2 \Delta_{m}$, the $n$th central moment is given by

$$
\begin{aligned}
M_{n m}\left(\theta_{0 m}\right) & =\int_{\theta_{0 m}-\Delta_{m}}^{\theta_{0 m}+\Delta_{m}} \frac{\left(\theta-\theta_{0 m}\right)^{n}}{2 \Delta_{m}} d \theta \\
& = \begin{cases}\frac{\Delta_{m}^{n}}{n+1}, & n \text { is even } \\
0, & n \text { is odd. }\end{cases}
\end{aligned}
$$

For a Gaussian ID (GID) source with the central angle $\theta_{0 m}$ and the angular spread $2 \Delta_{m}$, the $n$th central moment can be expressed as [32]

$$
M_{n m}\left(\theta_{0 m}\right)=\left\{\begin{array}{cc}
1 \cdot 3 \cdot 5 \cdots(n-1) \Delta_{m}^{n}, & n \text { is even } \\
0, & n \text { is odd }
\end{array}\right.
$$

For a Laplacian ID (LID) source with the central angle $\theta_{0 m}$ and the angular spread $2 \Delta_{m}$, the expression for the $n$th central moment takes the following form:

$$
M_{n m}\left(\theta_{0 m}\right)=\left\{\begin{array}{cl}
n ! 2^{-n / 2} \Delta_{m}^{n}, & n \text { is even } \\
0, & n \text { is odd }
\end{array}\right.
$$

Hence, having one of the even-indexed central moments and assuming a certain parametric angular power density, we can estimate the angular spread. For example, using the estimate of the second central moment, we have that for UID sources

$$
\hat{\Delta}_{m}=\sqrt{3 \hat{M}_{2 m}\left(\hat{\theta}_{0 m}\right)}
$$

whereas for GID/LID sources

$$
\hat{\Delta}_{m}=\sqrt{\hat{M}_{2 m}\left(\hat{\theta}_{0 m}\right)} \text {. }
$$

It is worth noting that the estimates of the source central angles can be refined by an iterative algorithm in which the estimates of the source central angles are used in (37) instead of $\tilde{\theta}_{0 m}$, $m=1,2, \ldots, q$. This refinement procedure can be iterated a few times to improve the estimates.
Now, we can summarize our algorithm as follows.

Step 1) Compute the sample covariance matrix $\hat{\boldsymbol{R}}_{x x}$, and specify the initial values of $\tilde{\theta}_{0 m}, m=1,2, \ldots, q$.

Step 2) Compute $\hat{\boldsymbol{m}}\left(\tilde{\boldsymbol{\theta}}_{0}\right)$ from (37) and, using (24) and (25), find the estimates $\hat{M}_{1 m}\left(\tilde{\theta}_{0 m}\right), m=1,2, \ldots, q$ from the proper elements of the vector $\hat{\boldsymbol{m}}\left(\tilde{\boldsymbol{\theta}}_{0}\right)$.

Step 3) Update $\tilde{\theta}_{0 m}=\tilde{\theta}_{0 m}+\hat{M}_{1 m}\left(\tilde{\theta}_{0 m}\right)$, and set $\hat{\theta}_{0 m}=$ $\tilde{\theta}_{0 m}$.

Step 4) Repeat steps 2 and 3 a few times. Compute $\boldsymbol{Q}\left(\hat{\boldsymbol{\theta}}_{0}\right)$ and $\boldsymbol{p}\left(\hat{\boldsymbol{\theta}}_{0}\right)$ from (31) and (32), respectively. Then, using (37), calculate the vector $\hat{\boldsymbol{m}}\left(\hat{\boldsymbol{\theta}}_{0}\right)$, and obtain $\hat{M}_{2 m}\left(\hat{\theta}_{0 m}\right)$ from the proper elements of this vector.

Step 5) Estimate the source angular spread from the previously estimated second central moments $\hat{M}_{2 m}\left(\hat{\theta}_{0 m}\right), m=1,2, \ldots, q$ [for example, use (42) and (43) for the UID and GID/LID sources, respectively].

Remark 1: The algorithm is initialized using the preliminary (coarse) estimates of the source DOAs. Note that a priori knowledge of either angular sectors or initial estimates of the signal DOAs is required in numerous array processing algorithms; see [33]-[44]. Such preliminary estimates can be easily obtained using the conventional beamformer [22], [37].

Remark 2: It is worth noting that the knowledge of the shape of the angular power density function is required only in the last step of our algorithm. Therefore, if this knowledge is not available, one can use the estimated second central moments as a measure of the source angular spread parameters. In addition, it is important to stress that the central angle estimates do not depend on the shape of the angular power density function. Furthermore, according to (43), the angular spread estimates are not affected by mismodeling GID sources as LID sources or vice versa. Therefore, our estimator is quite insensitive to uncertainties in the shape of the angular power density function.

\section{Simulation Results}

In our simulations, we assume a ULA of $p=11$ omnidirectional sensors spaced half a wavelength apart. The number of statistically independent snapshots in each simulation run is $N=500$. A total of 100 independent simulation runs are performed to obtain each simulated point. Note that by increasing the parameter $I$, we are able to improve the quality of approximation of the covariance matrix. However, at the same time, the sensitivity of the algorithm to array calibration errors increases in this case (i.e., $I$ should be not much larger than one). Because of this fact, we have chosen to implement our algorithm with $I=3$. Using extensive simulations with different scenarios, we have found that our algorithm converges in less than four iterations. Therefore, three iterations of steps 2 and 3 are used in all our examples. All initial values of $\tilde{\theta}_{0 m}, m=1, \ldots, q$ have been chosen far enough from the true central angles so that the difference between these initial values and the true central angles is larger than that between the true central angles and their estimates obtained by means of conventional beamformer.

In the first example, we model the case of a single distributed source. This source is assumed to be either UID or GID with the central angle $\theta_{0}=0^{\circ}$ and the angular spread $2 \Delta=4^{\circ}$. In this 


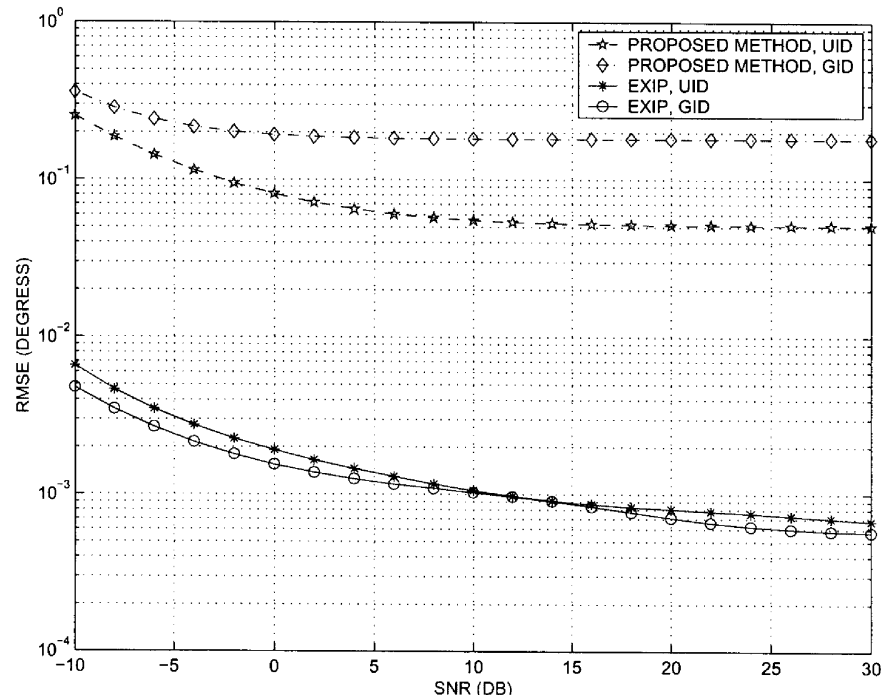

Fig. 1. RMSE of the central angle estimates versus the SNR; first example.

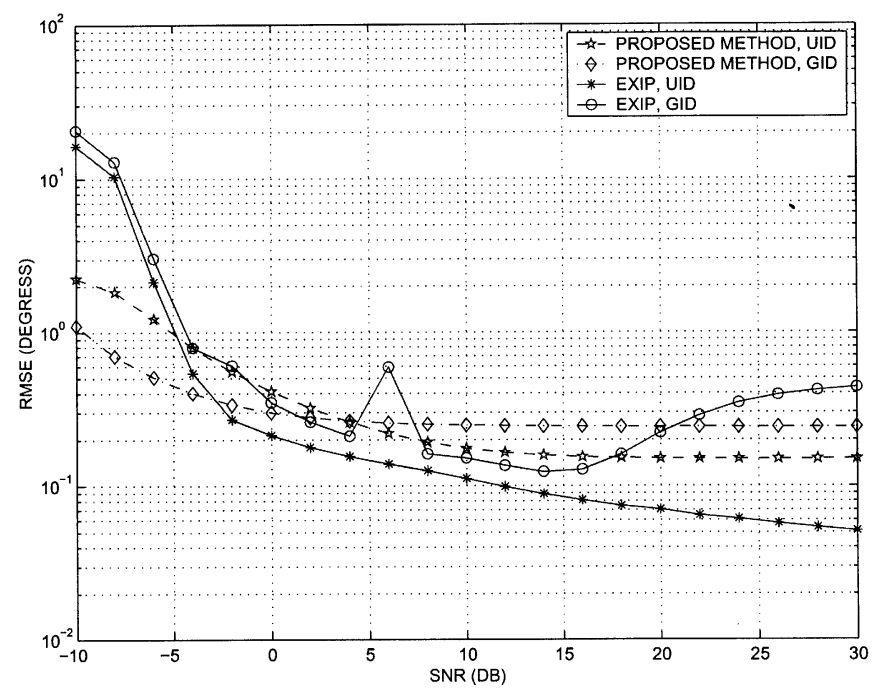

Fig. 2. RMSE of the angular spread estimates versus the SNR; first example.

example, we compare the performances of our algorithm (with the initial value $\tilde{\theta}_{0}=5^{\circ}$ ) and the EXIP method. ${ }^{4}$

Figs. 1 and 2 show the root-mean-squared errors (RMSEs) of the estimates of the central angle and angular spread, respectively, versus the SNR. From Fig. 1, we see that in terms of central angle estimation, EXIP has a substantially better performance than the algorithm proposed. Indeed, our technique gives biased estimates even at high values of SNR. However, as follows from Fig. 2, the performances of the algorithms tested are comparable in terms of angular spread estimation. In particular, at high SNRs in Fig. 2, the EXIP method has a visibly better performance than our method in the UID source scenario, but its performance in the GID scenario is worse than that of the proposed technique. One of possible explanations of this fact is that the EXIP estimator takes no advantage of knowing the form of the angular distribution function, whereas our method does.

${ }^{4}$ Note that the EXIP algorithm has been developed for the single source case only. For this case, the EXIP approach has been shown to be one of the best methods of choice [21].

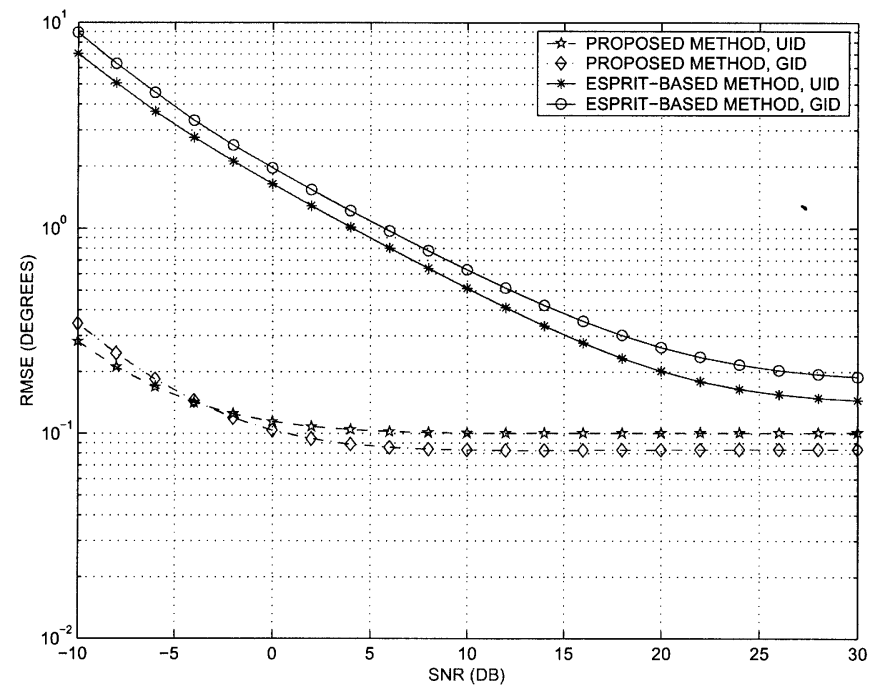

Fig. 3. RMSE of the central angle estimates versus the SNR; second example.

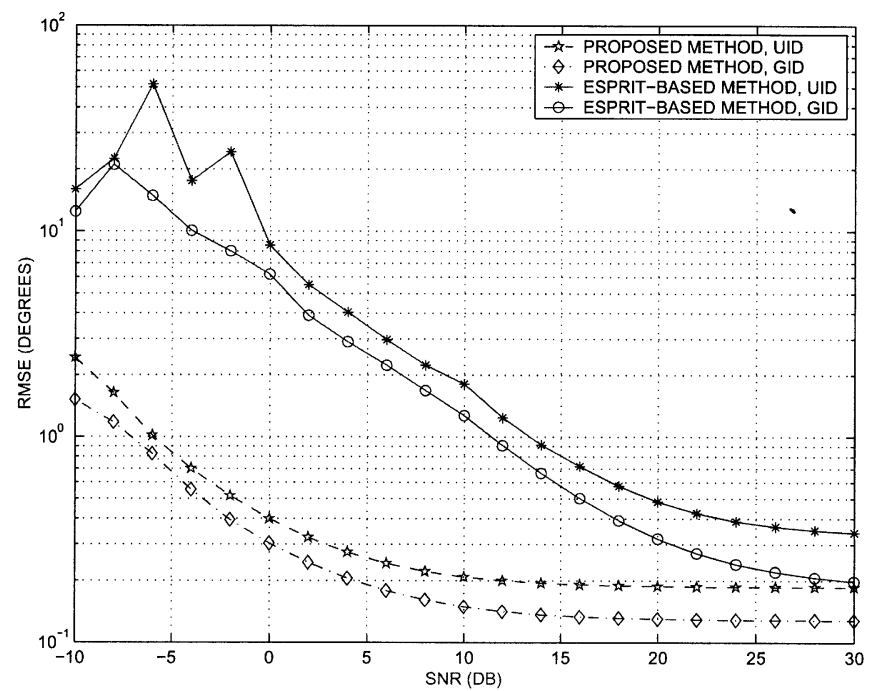

Fig. 4. RMSE of the angular spread estimates versus the SNR; second example.

Note that essential advantages of the proposed algorithm relative to EXIP are that our technique has lower computational cost and that its application is not restricted to the single-source case.

In the second example, we assume two distributed sources. One of them is UID with the central angle $\theta_{01}=10^{\circ}$ and the angular spread $2 \Delta_{1}=5^{\circ}$. The second source is GID with the central angle $\theta_{02}=30^{\circ}$ and the angular spread $2 \Delta_{2}=3^{\circ}$. In this example, we compare our method (with the initial values $\tilde{\theta}_{01}=$ $5^{\circ}$ and $\tilde{\theta}_{02}=35^{\circ}$ ) with the ESPRIT-based method [23]. To simulate the ESPRIT-based algorithm in a proper way, two identical 11-element ULAs with the half-wavelength interelement spacing have been assumed, and the intersubarray displacement $\lambda / 10$ has been chosen, where $\lambda$ is the wavelength. ${ }^{5}$ Figs. 3 and 4 display the RMSEs of the estimates of the central angle and angular spread, respectively, versus the SNR. From these figures, we see that our method essentially outperforms the ESPRIT-

${ }^{5}$ Note that the ESPRIT-based algorithm requires rather small inter-subarray displacement to to reduce the bias [23]. 


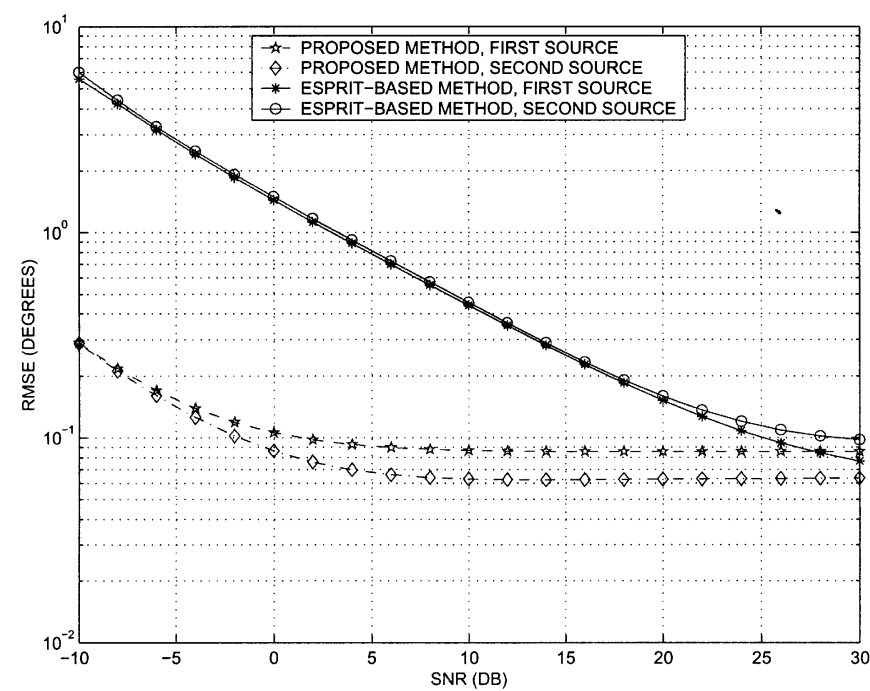

Fig. 5. RMSE of the central angle estimates versus the SNR; third example.

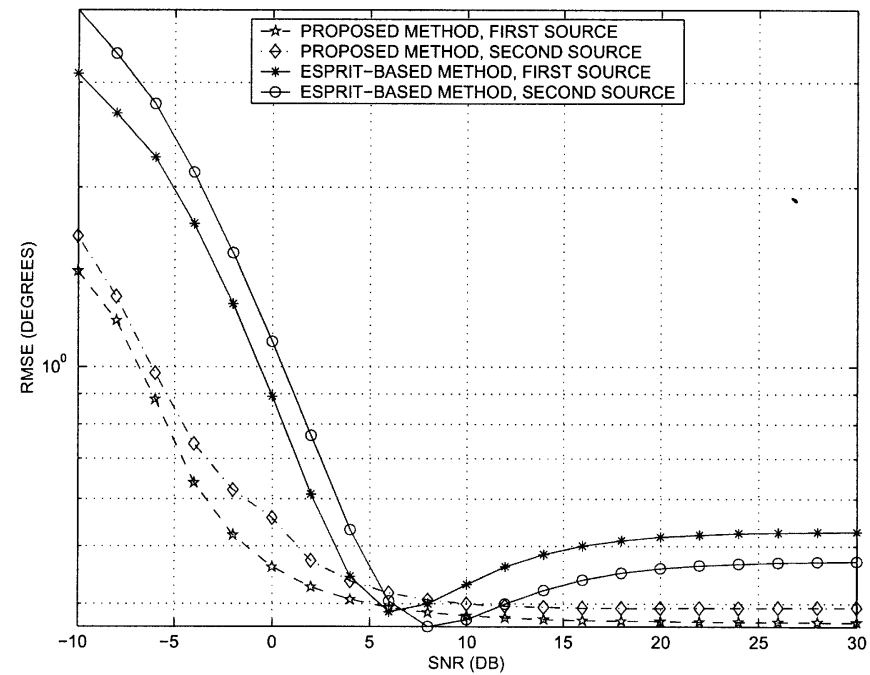

Fig. 6. RMSE of the angular spread estimates versus the SNR; third example.

based approach. In particular, it can be seen that the ESPRITbased algorithm has much higher SNR threshold both in central angle and angular spread estimates than the proposed technique. Interestingly, the performance of our algorithm is better in the GID case, whereas the ESPRIT-based method shows the opposite effect. The explanation of this fact is that these two methods use different approaches to estimate the source central angles. In particular, the ESPRIT-type algorithm approximates the distributed source with two point sources, whereas our technique uses the Taylor series for this purpose.

In our third example, we consider the case of two LID sources with the central angles $\theta_{01}=10^{\circ}$ and $\theta_{02}=30^{\circ}$ and the angular spreads $2 \Delta_{1}=2 \Delta_{2}=2.8^{\circ}$, respectively. Similar to the previous example, our method (with the initial values $\tilde{\theta}_{01}=5^{\circ}$ and $\tilde{\theta}_{02}=35^{\circ}$ ) and the ESPRIT-based algorithm are compared. Figs. 5 and 6 display the RMSEs of the estimates of the central angle and the angular spread, respectively, versus the SNR. Similar to the previous example, we observe that the proposed method drastically outperforms the ESPRIT-based estimator. It

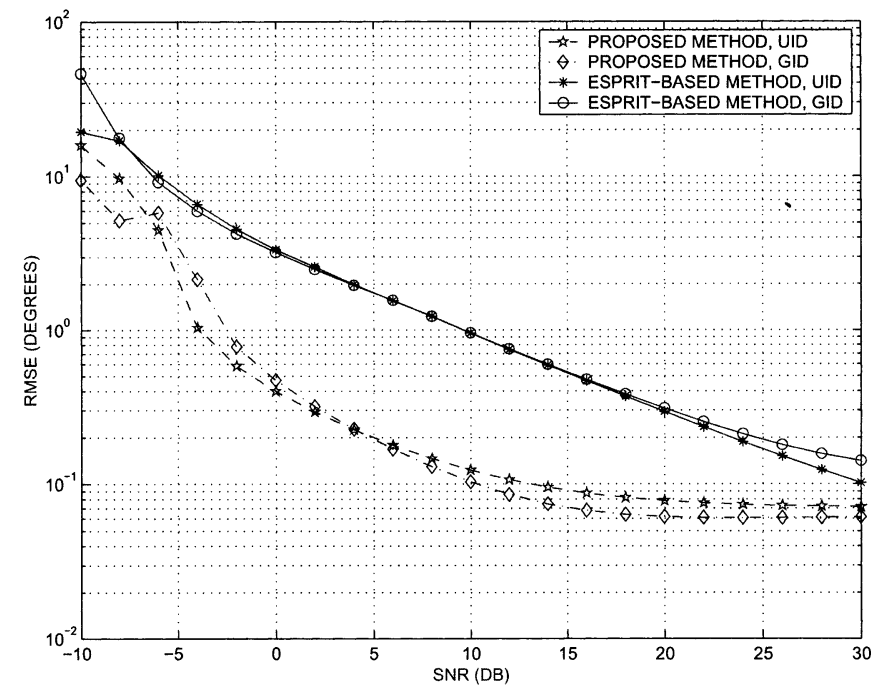

Fig. 7. RMSE of the central angle estimates versus the SNR; fourth example.

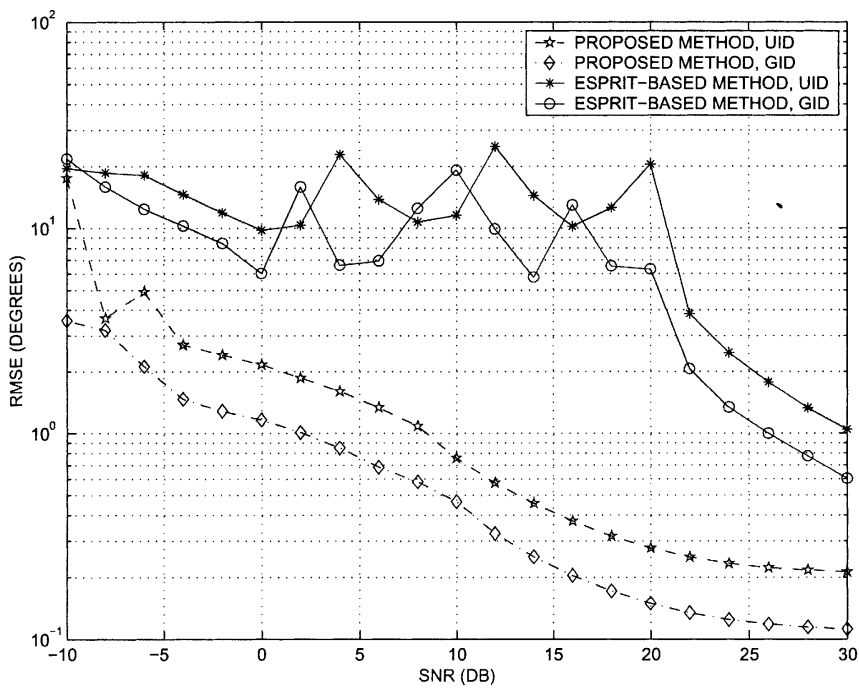

Fig. 8. RMSE of the angular spread estimates versus the SNR; fourth example.

can be seen from Fig. 5 that in this example, the ESPRIT-based method has much higher SNR threshold than the proposed technique. As it follows from Fig. 6, the angular spread estimation performance of the ESPRIT-based method does not decrease monotonically with SNR because of strongly biased estimates. At the same time, the proposed estimator can be seen to have much smaller bias, and its angular spread estimation performance decreases monotonically with SNR.

In our last example, we consider the case of two sources, but, in contrast to the previous two examples, these sources are closely spaced. The first source was modeled as a UID source with the central angle $\theta_{01}=10^{\circ}$ and angular spread $2 \Delta_{1}=$ $3^{\circ}$, whereas the second source was GID with the central angle $\theta_{02}=17^{\circ}$ and angular spread $2 \Delta_{2}=2^{\circ}$. Again, we compare our method (with the initial values $\tilde{\theta}_{01}=5^{\circ}$ and $\tilde{\theta}_{02}=22^{\circ}$ ) and the ESPRIT-based algorithm. Figs. 7 and 8 show the RMSEs of the estimates of the central angle and the angular spread, respectively, versus the SNR. Similar to the previous two examples, 
our technique substantially outperforms the ESPRIT-based algorithm in having much lower SNR threshold for both central angle and angular spread estimates than the proposed technique. By comparing Figs. 8 and 4, we see that for closely spaced sources, this effect is stronger than for widely spaced sources.

We conclude this section with two comments on other simulation results that are not included in the manuscript in the interest of brevity.

As any other parametric method, our method breaks down if the number of sources is underestimated. However, it is very robust to overestimating this number. In the latter case, the estimated power (the estimate of the first central moment) is close to zero for all spurious sources, and this helps to finally determine the true number of sources and their parameter estimates.

Another question we have studied is whether the performance of our method breaks down in scenarios where, apart from spread sources, point sources are present. Our simulations have shown that the proposed algorithm is applicable to such scenarios without affecting its performance.

\section{CONCLUSIONS}

We have presented a new parametric approach to localization of multiple incoherently distributed sources in sensor array. Our algorithm approximates the covariance matrix using central and noncentral moments of the source angular power densities. Based on this approximation, a simple covariance fitting optimization technique is proposed to estimate these moments. Then, the source parameters are obtained from the moment estimates. Compared to the existing methods, our approach has a reduced computational cost and is applicable to the multisource scenarios with different angular power densities.

\section{ACKNOWLEDGMENT}

The authors are grateful to Prof. O. Besson, who provided the access to his MATLAB code for the EXIP algorithm. The authors also wish to thank Dr. M. Bengtsson for his useful comments on the earlier version of the manuscript and one of anonymous reviewers, whose comments helped us to simplify our derivations in Section III.

\section{REFERENCES}

[1] R. O. Schmidt, "Multiple emitter location and signal parameter estimation," in Proc. RADC Spectral Estim. Workshop, Rome, NY, 1979, pp. 234-258.

[2] R. Roy and T. Kailath, "ESPRIT-Estimation of signal parameters via rotational invariance techniques," IEEE Trans. Acoust., Speech, Signal Processing, vol. 37, pp. 984-995, July 1989.

[3] P. Zetterberg, "Mobile cellular communications with base station antenna arrays: Spectrum efficiency, algorithms, and propagation models," Ph.D. dissertation, Royal Inst. Technol., Stockholm, Sweden, 1997.

[4] K. I. Pedersen, P. E. Mogensen, and B. H. Fleury, "Analysis of time, azimuth and Doppler dispersion in outdoor radio channels," in Proc. ACTS Mobile Commun. Summit, Aalborg, Denmark, Oct. 1997, pp. 308-313.

[5] D. Astely, "Spatio and spatio-temporal processing with antenna arrays in wireless systems," Ph.D. dissertation, Royal Inst. Technol., Stockholm, Sweden, 1999.

[6] K. I. Pedersen, P. E. Mogensen, and B. H. Fleury, "Spatial channel characteristics in outdoor environment and their impact on BS antenna system performance," in Proc. IEEE Veh. Technol. Conf., Ottawa, ON, Canada, May 1998, pp. 719-724.
[7] L. C. Godara, "Application of antenna arrays to mobile communications-Part I: Performance improvement, feasibility, and system considerations," Proc. IEEE, vol. 85, pp. 1031-1060, July 1997.

[8] _ , "Application of antenna arrays to mobile communications-Part II: Beamforming and direction-of-arrival considerations," Proc. IEEE, vol. 85, pp. 1195-1245, Aug. 1997.

[9] A. J. Paulraj and C. B. Papadias, "Space-time processing for wireless communications," IEEE Signal Processing Mag., vol. 14, pp. 49-83, Nov. 1997.

[10] R. Raich, J. Goldberg, and H. Messer, "Bearing estimation for a distributed source: Modeling, inherent accuracy limitations and algorithms," IEEE Trans. Signal Processing, vol. 48, pp. 429-441, Feb. 2000.

[11] S. Valaee, B. Champagne, and P. Kabal, "Parametric localization of distributed sources," IEEE Trans. Signal Processing, vol. 43, pp. 2144-2153, Sept. 1995.

[12] Y. Meng, P. Stoica, and K. M. Wong, "Estimation of the directions of arrival of spatially dispersed signals in array processing," Proc. Inst. Elect. Eng.-Radar, Sonar Navigat., vol. 143, pp. 1-9, Feb. 1996.

[13] M. Bengtsson, "Antenna array processing for high rank data models," Ph.D. dissertation, Royal Inst. Technol., Stockholm, Sweden, 1999.

[14] T. Trump and B. Ottersten, "Estimation of nominal direction of arrival and angular spread using an array of sensors," Signal Process., vol. 50, pp. 57-69, Apr. 1996.

[15] S. Shahbazpanahi, S. Valaee, and M. Nayebi, "Distributed source parameter estimation," in Proc. 5th Iran. Conf. Elect. Eng., May 1997, pp. 6/304-6/311.

[16] S. Shahbazpanahi, S. Valaee, B. Champagne, and P. Kabal, "Extended source localization using the ESPRIT algorithm," in Proc. ICT, Apr. 1997, pp. 1033-1037

[17] M. Bengtsson and B. Ottersten, "Low-complexity estimators for distributed sources," IEEE Trans. Signal Processing, vol. 48, pp. 2185-2194, Aug. 2000.

[18] O. Besson and P. Stoica, "A fast and robust algorithm for DOA estimation of a spatially dispersed source," Digital Signal Process., vol. 9, pp. 267-279, 1999.

[19] O. Besson, F. Vincent, P. Stoica, and A. B. Gershman, "Approximate maximum likelihood estimators for array processing in multiplicative noise environments," IEEE Trans. Signal Processing, vol. 48, pp. 2506-2518, Sept. 2000.

[20] O. Besson, P. Stoica, and A. B. Gershman, "A simple and accurate direction of arrival estimator in the case of imperfect spatial coherence," IEEE Trans. Signal Processing, vol. 49, pp. 730-737, Apr. 2001.

[21] O. Besson and P. Stoica, "Decoupled estimation of DOA and angular spread for a spatially distributed source," IEEE Trans. Signal Processing, vol. 48, pp. 1872-1882, July 2000.

[22] R. Raviv, J. Goldberg, and H. Messer, "Bearing estimation for a distributed source via the conventional beamformer," in Proc. 9th IEEE Workshop Stat. Signal Array Process., 1998, pp. 5-8.

[23] S. Shahbazpanahi, S. Valaee, and M. H. Bastani, "Distributed source localization using ESPRIT algorithm," IEEE Trans. Signal Processing, vol. 49, pp. 2169-2178, Oct. 2001.

[24] M. Bengtsson and B. Volcker, "On the estimation of azimuth distributions and azimuth spectra," in Proc. Veh. Technol. Conf., vol. 3, Atlantic City, NJ, Oct. 2001, pp. 1612-1615.

[25] A. B. Gershman, C. F. Mecklenbräuker, and J. F. Böhme, "Matrix fitting approach to direction of arrival estimation with imperfect spatial coherence of wavefronts," IEEE Trans. Signal Processing, vol. 45, pp. 1894-1899, July 1997.

[26] B. Ottersten, P. Stoica, and R. Roy, "Covariance matching estimation techniques for array signal processing applications," Digital Signal Processing, vol. 8, pp. 185-208, July 1998.

[27] J. Ringelstein, A. B. Gershman, and J. F. Böhme, "Direction finding in random inhomogeneous media in the presence of multiplicative noise," IEEE Signal Processing Lett., vol. 7, pp. 269-271, Oct. 2000.

[28] M. Ghogho, O. Besson, and A. Swami, "Estimation of directions of arrival of multiple scattered sources," IEEE Trans. Signal Processing, vol. 49, pp. 3467-3480, Nov. 2001.

[29] A. B. Gershman, U. Nickel, and J. F. Böhme, "Adaptive beamforming algorithms with robustness against jammer motion," IEEE Trans. Signal Processing, vol. 45, pp. 1878-1885, July 1997.

[30] S. D. Hayward, "Effects of motion on adaptive arrays," Proc. Inst. Elect. Eng.-Radar, Sonar, Navigat., vol. 144, pp. 15-20, Feb. 1997.

[31] V. Katkovnik and A. B. Gershman, "Performance study of the local polynomial approximation based beamforming in the presence of moving sources," IEEE Trans. Antennas Propagat., vol. 50, pp. 1151-1157, Aug. 2002. 
[32] A. Papoulis, Probability, Random Variables, and Stochastic Processes, Third ed. New York: McGraw-Hill, 1991.

[33] H. Wang and M. Kaveh, "Coherent signal-subspace processing for the detection and estimation of angles of arrival of multiple wide-band sources," IEEE Trans. Acoust., Speech, Signal Processing, vol. ASSP-33, pp. 823-831, Aug. 1985.

[34] K. M. Buckley and X.-L. Xu, "Spatial-spectrum estimation in a location sector," IEEE Trans. Acoust., Speech., Signal Processing, vol. 38, pp. 1842-1852, Nov. 1990.

[35] R. J. Kozick and S. A. Kassam, "A unified approach to coherent source decorrelation by autocorrelation matrix smoothing," Signal Process., vol. 45, pp. 115-130, July 1995.

[36] M. D. Zoltowski, G. M. Kautz, and S. D. Silverstein, "Beamspace rootMUSIC," IEEE Trans. Signal Processing, vol. 41, pp. 344-364, Jan. 1993.

[37] A. B. Gershman, "Pseudo-randomly generated estimator banks: A new tool for improving the threshold performance of direction finding," IEEE Trans. Signal Processing, vol. 46, pp. 1351-1364, May 1998.

[38] — "Direction finding using beamspace root estimator banks," IEEE Trans. Signal Processing, vol. 46, pp. 3131-3135, Nov. 1998.

[39] S. Anderson, "On optimal dimension reduction for sensor array signal processing," Signal Process., vol. 30, pp. 245-256, Jan. 1993.

[40] B. Friedlander, "The root-MUSIC algorithm for direction finding with interpolated arrays," Signal Process., vol. 30, pp. 15-25, 1993.

[41] D. V. Sidorovich and A. B. Gershman, "2-D wideband interpolated rootMUSIC applied to measured seismic data," IEEE Trans. Signal Processing, vol. 46, pp. 2263-2267, Aug. 1998

[42] A. B. Gershman and H. Messer, "Robust mixed-order root-MUSIC," Circuits, Syst., Signal Process., vol. 19, no. 5, pp. 451-466, Oct. 2000.

[43] M. Agrawal and S. Prasad, "A modified likelihood function approach to DOA estimation in the presence of unknown spatially correlated gaussian noise using a uniform linear array," IEEE Trans. Signal Processing, vol. 48, pp. 2743-2749, Oct. 2000

[44] A. B. Gershman and M. G. Amin, "Wideband direction-of-arrival estimation of multiple chirp signals using spatial time-frequency distributions," IEEE Signal Processing Lett., vol. 7, pp. 152-155, June 2000.

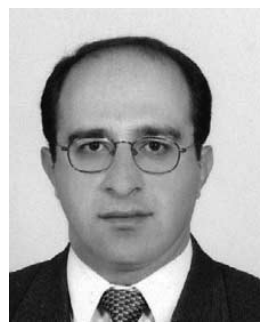

Shahram Shahbazpanahi (M'02) was born in Sanandaj, Kurdistan, Iran. He received the B.Sc., M.Sc., and Ph.D. degrees from Sharif University of Technology, Tehran, Iran, in 1992, 1994, and 2001, respectively, all in electrical engineering.

From September 1994 to September 1996, he was a Faculty Member with the Department of Electrical Engineering, Razi University, Kermanshah, Iran. Since July 2001, he has been conducting research as a Postdoctoral Fellow at the Department of Electrical and Computer Engineering, McMaster University, Hamilton, ON, Canada. From March to September 2002, he was a visiting researcher with the Department of Communication Systems, Gerhard-Mercator University, Duisburg, Germany. His research interests include statistical and array signal processing, space-time adaptive processing, detection and estimation, smart antennas, spread spectrum techniques, and DSP programming and hardware/real-time software design for telecommunication systems.

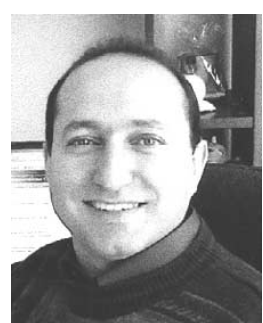

Shahrokh Valaee (S'88-M'00-SM'02) was born in Tabriz, Iran. He received the B.Sc. and M.Sc. degrees from Tehran University, Tehran, Iran, and the Ph.D. degree from McGill University, Montreal, QC, Canada, all in electrical engineering.

From 1994 to 1995, he was a Research Associate at INRS Telecom, University of Quebec, Montreal. From 1996 to 2001, he was an Assistant Professor with the Department of Electrical Engineering, Tarbiat Modares University, Tehran, and an Adjunct Professor with the Department of Electrical Engineering, Sharif University of Technology, Tehran. Since September 2001, he has been an Associate Professor with the Edward S. Rogers, Sr. Department of Electrical and Computer Engineering, University of Toronto, Toronto, ON, Canada. His current research interests are in wireless $a d$ hoc and cellular networks, quality-ofservice provisioning in high-speed networks, and distributed signal localization.

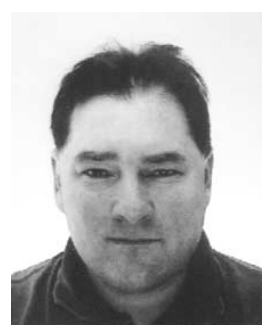

Alex B. Gershman (M'97-SM'98) received the Diploma (M.S.) and Ph.D. degrees in radiophysics from the Nizhny Novgorod State University, Nizhny Novgorod, Russia, in 1984 and 1990, respectively.

From 1984 to 1989 , he was with the Radiotechnical and Radiophysical Institutes, Nizhny Novgorod. From 1989 to 1997, he was with the Institute of Applied Physics, Russian Academy of Science, Nizhny Novgorod, as a Senior Research Scientist. From the summer of 1994 until the beginning of 1995, he was a Visiting Research Fellow at the Swiss Federal Institute of Technology, Lausanne, Switzerland. From 1995 to 1997, he was Alexander von Humboldt Fellow at Ruhr University, Bochum, Germany. From 1997 to 1999, he was a Research Associate at the Department of Electrical Engineering, Ruhr University. In 1999, he joined the Department of Electrical and Computer Engineering, McMaster University, Hamilton, ON, as an Associate Professor, where he became a Full Professor in 2002. Currently, he also holds a Visiting Professorship at the Department of Communication Systems, Gerhard-Mercator University, Duisburg, Germany, and Fraunhofer Institute, Duisburg. His research interests are in the area of signal processing and include robust statistical signal and array processing, adaptive beamforming and smart antennas for mobile communications, signal parameter estimation and detection, spectral analysis, and signal processing applications to wireless communications, underwater acoustics, seismology, and radar.

Dr. Gershman was a recipient of the 1993 International Union of Radio Science (URSI) Young Scientist Award, the 1994 Outstanding Young Scientist Presidential Fellowship (Russia), the 1994 Swiss Academy of Engineering Science and Branco Weiss Fellowships (Switzerland), and the 1995-1996 Alexander von Humboldt Fellowship (Germany). He received the 2000 Premier's Research Excellence Award of Ontario, Canada, and the 2001 Wolfgang Paul Award from the Alexander von Humboldt Foundation, Germany. He was also a recipient of the 2002 Young Explorers Prize from the Canadian Institute for Advanced Research (CIAR), which honors Canada's top 20 researchers aged 40 or under. Since 1999, he has been an Associate Editor of IEEE TRANSACTIONS ON Signal PROCESSING and a Member of the Sensor Array and Multichannel (SAM) Signal Processing Technical Committee of the IEEE Signal Processing Society. 